\title{
Experimental analysis on Rowe's stress-dilatancy relation and frictional instability of fault gouges
}

Department of Earth Science, Tohoku University, 6-3, Aramaki Aza-Aoba, Aoba-ku, Sendai, Miyagi, 980-8578, Japan. E-mail: mhirata@dc.tohoku.ac.jp

The stress-dilatancy relation is important for understanding fault mechanics and brittle deformation of rocks, and hence the onset of frictional instability. The principle of minimum energy ratio was proposed by Rowe, and this Rowe's theory has been applied to deformation of granular materials. Rowe suggested that the energy ratio, which is the ratio of the energy dissipation rate to energy supply rate, would be a minimum and constant value. The relation between the rate of dilatancy and the maximum stress ratio can be extended to the case of a random assembly of irregular particles whereby the rate of internal work absorbed in frictional heat is a minimum as the mass dilates. According to Rowe's law, experiments show that the minimum energy criterion is closely obeyed by highly dilatant dense over-consolidated and reloaded assemblies throughout deformation. However, it is unknown whether or not the principle of minimum energy ratio can be applied to fault mechanics. The results of our friction experiments show the possibility that the energy ratio can be a new factor for evaluating frictional instability.

\section{Introduction}

Reynolds (1885) showed that dense sands expand at failure, whereas loose sands contract during shear failure. Therefore, because dilatancy is generally observed as a precursor to brittle faulting and the development of shear localization, attention has been focused on how localized failure develops in a dilatant rock (Wong et al., 2001). Dilatancy is hence closely related to earthquakes, and so to clarify the stress-dilatancy relationship is very important from the point of disaster prevention. Based on stress-dilatancy relation, the principle of minimum energy ratio was introduced by Rowe (1962) to obtain a stress-dilatancy relation for granular materials in a dense packing state under axisymmetric stress conditions. Rowe (1962) postulated that the ratio of energy dissipation by internal friction to energy supply in the direction of the major principal stress would be a constant and minimum value. The Rowe's theory can be applied not only to granular materials such as fault gouges but also to blocky materials such as lithified rocks. Niiseki (2001) and Moroto (1991) discussed Rowe's law in terms of thermodynamics and statistical mechanics of granular materials. However, there are many questions about Rowe's law, for instance, the physical meaning of an internal friction angle which is the factor of the energy ratio. Hence, it has not been utilized much for fault mechanics until now. Chappell (1979) proposed a relationship between the principal stresses in terms of the joint friction angle, whose magnitude is not only dependent on the smooth joint friction angle and roughness but also on the rotation of the blocks caused by slip.

Moreover, natural faults produce gouge due to slip, that plays an important role in controlling the stability of natural faults (e.g., Scholz et al., 1969; Marone and Scholz, 1988). Therefore, an understanding of earthquake mechanics requires the knowledge of the frictional behavior of gouge and gouge-rock systems (Marone et al., 1990). Hence, many studies about gouge have clarified that frictional instability is correlated with microstructural development of gouge (e.g., Byerlee et al., 1978; Logan et al., 1979; Marone and Kilgore, 1993; Ikari et al., 2011; Onuma et al., 2011). These studies have shown the development of shear localization in a gouge layer and possibility that the development of shear localization influences the frictional instability. However, the relationship between microstructural development and frictional instability of gouge has not yet been clear from the perspective of Rowe's law. In this study, we conducted the friction experiments using the gouge in a gas-medium apparatus in order to test whether Rowe's theory of constant energy ratio holds for frictional instability of simulated fault gouge and to clarify these relationships based on Rowe's law. The results of our friction experiments reveal that the mechanical behavior of fault gouges obeys the Rowe's law. By this law, we will effectively be able to evaluate the longitudinal shape of a slip surface in landslides or spray fault in accretionary prisms and the potential occurrences of them.

\section{The friction experiments with a gas- medium apparatus}

The friction experiments using simulated fault gouge were conducted in a gas-medium apparatus under the confining pressures $\left(\mathrm{P}_{\mathrm{c}}\right)$ of 140-180 MPa (Figure 1). Strain rate was held constant at $10^{-3} / \mathrm{s}$. Dry quartz powders $(0.1$ or $0.2 \mathrm{~g})$ for gouge were sandwiched into two gabbroic forcing blocks ( $20 \mathrm{~mm}$ in diameter, $40 \mathrm{~mm}$ in length, and cut by a $50^{\circ}$ to their cylindrical axis). Three strain gauges glued onto a gouge layer through the Teflon jacket directly measured strain with the high-speed (sampling rate of $2 \mathrm{MHz}$ ) acquisition system (see Onuma et al., 2011 for details). To clarify mechanical behaviors of fault gouge, we held loads at different levels of differential stresses 


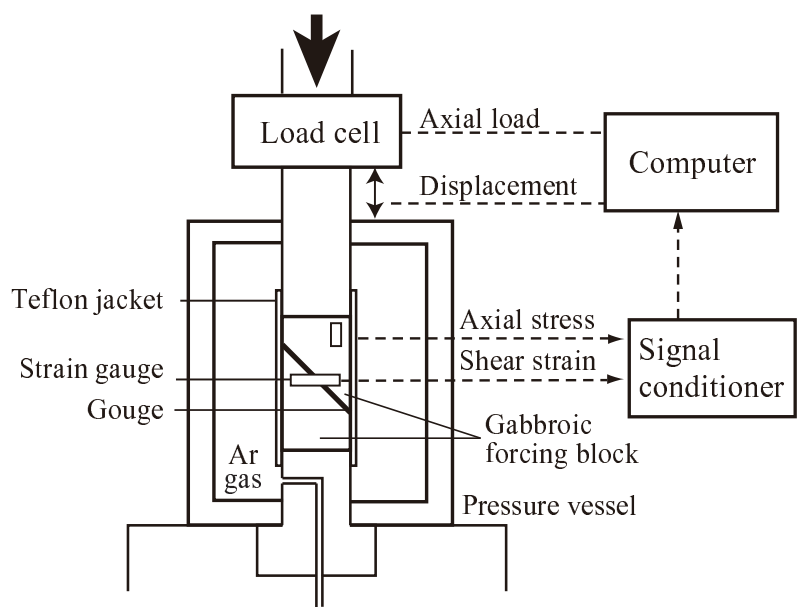

Figure 1. Schematic drawing of sample assembly and experimental setup.

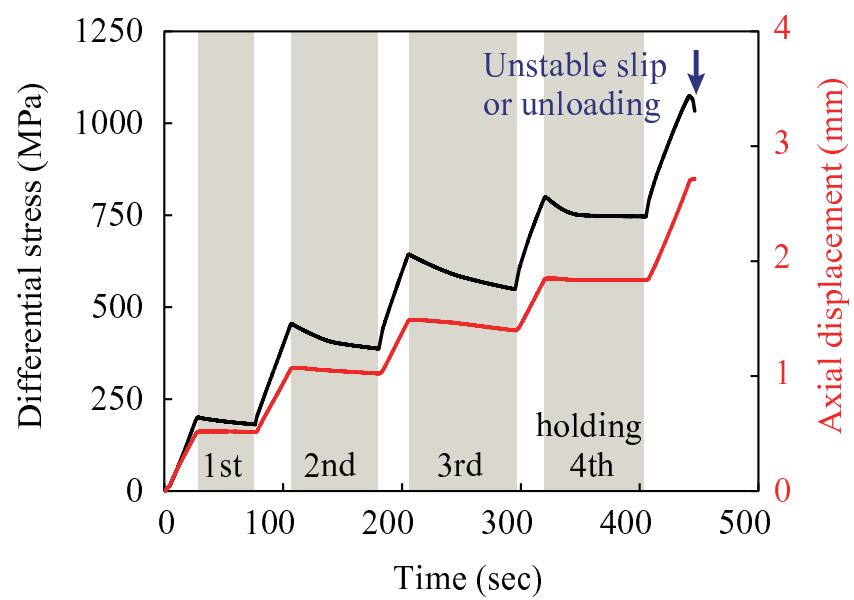

Figure 2. Operation of one cycle. After the 4th holding, the sample was loaded again until unstable slip occurred or strain gauges could not measure strains. When the latter happened, we unloaded and replaced to new strain gauges. Arrow means the end of one cycle. The gray shaded areas indicate the time ranges of holdings. The stress values are derived from the external load cell.

as shown in Figure 2 (1st holding at a differential stress of about 190 $\mathrm{MPa}, 2 \mathrm{nd}$ at $450 \mathrm{MPa}$, 3rd at $640 \mathrm{MPa}$, 4th at $800 \mathrm{MPa}$ ). When strains exceeded the measurable ranges of strain gauges, we replaced them with new ones. This succession of work, called cycle, was repeated until unstable slips occurred.

\section{Results}

We conducted 12 friction experiments at different confining pressures $P_{c}$ and different weights of gouges (Table 1). From our experimental data, the number of cycles up to unstable slip increased

Table 1. Experimental parameters. Cycle means the number of experiments until unstable slip occurred at each condition.

\begin{tabular}{ccc}
\hline $\mathrm{P}_{\mathrm{c}}(\mathrm{MPa})$ & Weight $(\mathrm{g})$ & Cycle \\
\hline 140 & 0.1 & 4 \\
160 & 0.1 & 3 \\
180 & 0.1 & 2 \\
180 & 0.2 & 3 \\
\hline
\end{tabular}

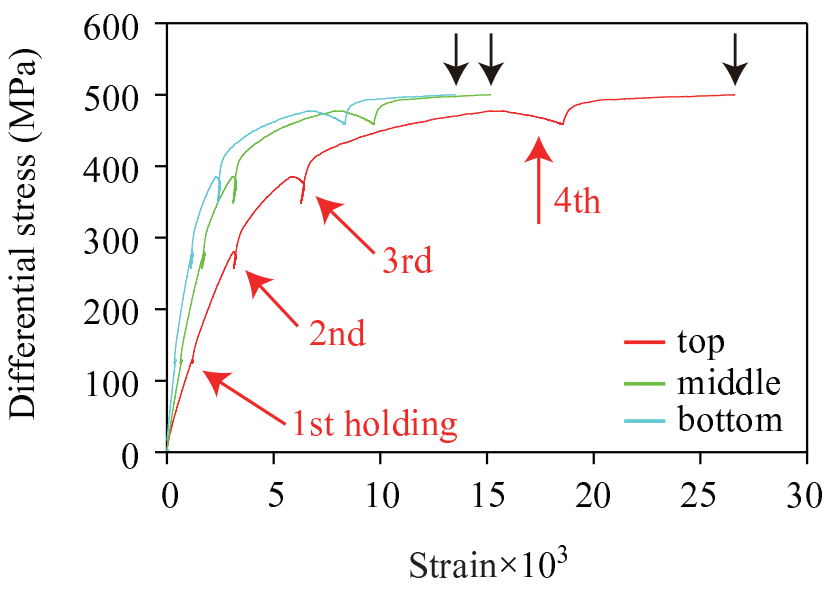

Figure 3. The representative stress-strain curve $\left(P_{c}: 140 \mathrm{MPa}\right.$, gouges: $0.1 \mathrm{~g}$, cycle 4). Top, middle and bottom mean position of strain gauges. Black arrow indicates unstable slip. The stress values are determined by the vertical strain gauge.

with decreasing confining pressures and increasing weight of gouges.

Representative stress-strain curves and the mechanical behaviors of gouges during holding stages are shown in Figure 3. The positive value of strain means extension. The color means position of strain gauges (Red: top strain gauge, green: middle, blue: bottom). We obtained three different behaviors of gouges depending on differential stresses determined by the vertical strain gauge: Contraction, transition from extension to contraction and great extension from low to high stresses (Figures 3 and 4). At 1st holding, so lower stress, gouges contracted in all experiments. The behaviors of gouges show transition at 2 nd or 3 rd holding, and great extension at 4 th holding. After repeated cycles, unstable slip took place at higher stress.

During holding stages, we also obtained the ratio of the energy rate of the major principal compressive stresses to minor compressive stresses, or the ratio of the input energy rate to output one. We obtained strain in the direction of minor principal compressive stress from three strain gauges glued onto a gouge layer. Similarly, the major principal compressive stress and strain were obtained from another gauge glued in vertical direction. The confining pressures were taken to be minor principal compressive stresses. The output energy can be expressed as a linear function of the input energy (Figure 5). This relation is expressed by

$$
\sigma_{1} \dot{\varepsilon}_{1}=K \sigma_{3} \dot{\varepsilon}_{3}
$$

where $K$ is the ratio of energy rates, $\sigma_{1}$ and $\sigma_{3}$ are the major and minor compressive stresses, and $\dot{\varepsilon}_{1}$ and $\dot{\varepsilon}_{3}$ are strain rates of each direction, respectively. Eq. (1) can be rewritten by

$$
\frac{\sigma_{1}}{\sigma_{3}}=K \frac{\dot{\varepsilon}_{3}}{\dot{\varepsilon}_{1}} .
$$

It is revealed that the energy ratio becomes large with confining pressure as shown in Figure 5. In this figure, each color means confining pressure (Yellow: $140 \mathrm{MPa}$, green: $160 \mathrm{MPa}$ and blue: $180 \mathrm{MPa}$ ). Moreover, because the energy ratio is slope of line, it is revealed that the energy ratio changed at each loading and holding stage (Figure 6). In Figure 6, the time passed to the direction from 

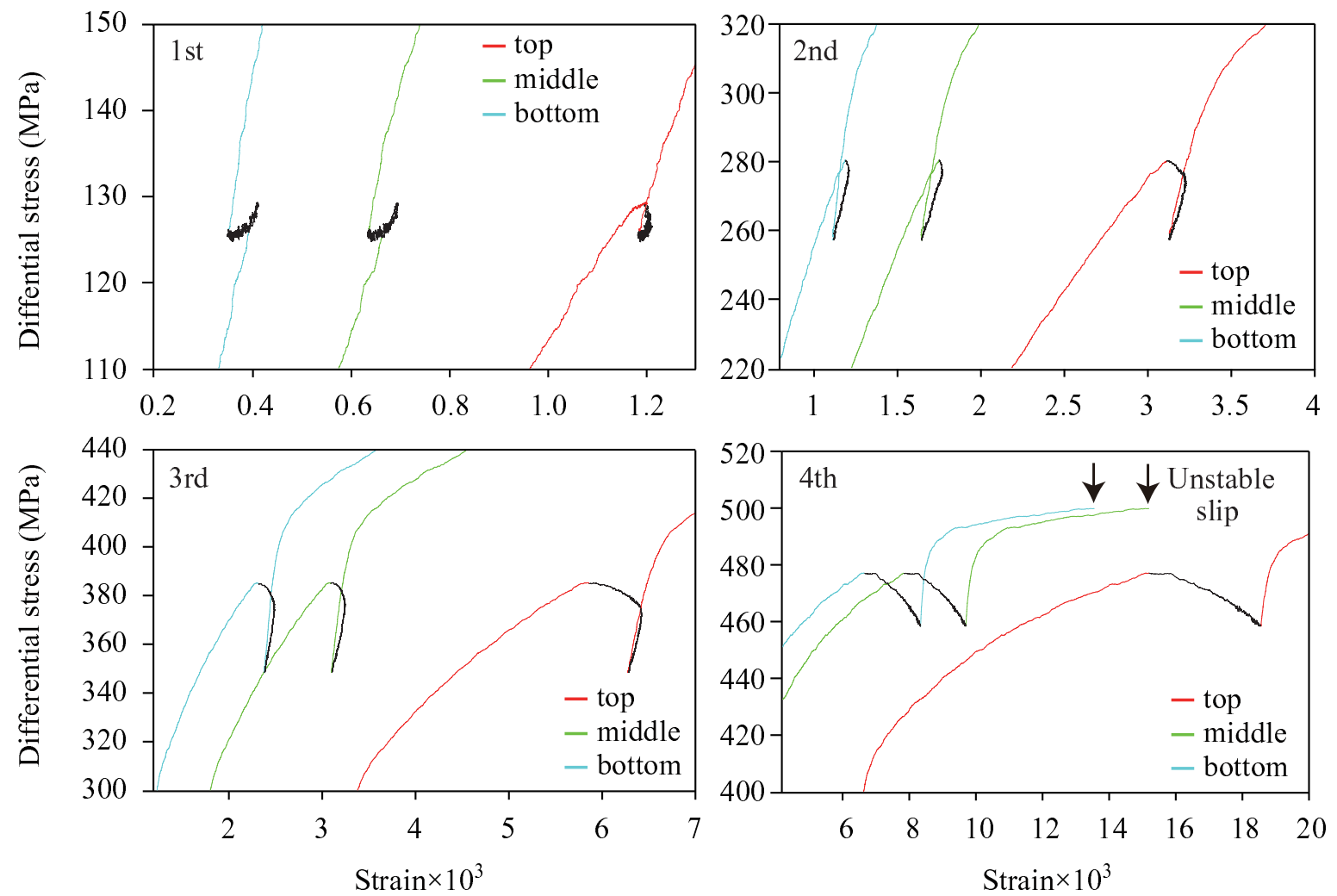

Figure 4. The enlarged diagram of Figure 3 at each holding period. Black lines mean the holding period.

the top right corner to the lower left until almost the 3rd holding stage. After that, Figure 6 shows the remarkable change in the energy ratio at the final loading stage.

\section{Discussion}

From our experiments, we consider that mechanical behaviors and the energy ratio of gouges are correlated with microstructural development. First, we discuss about the relationship between

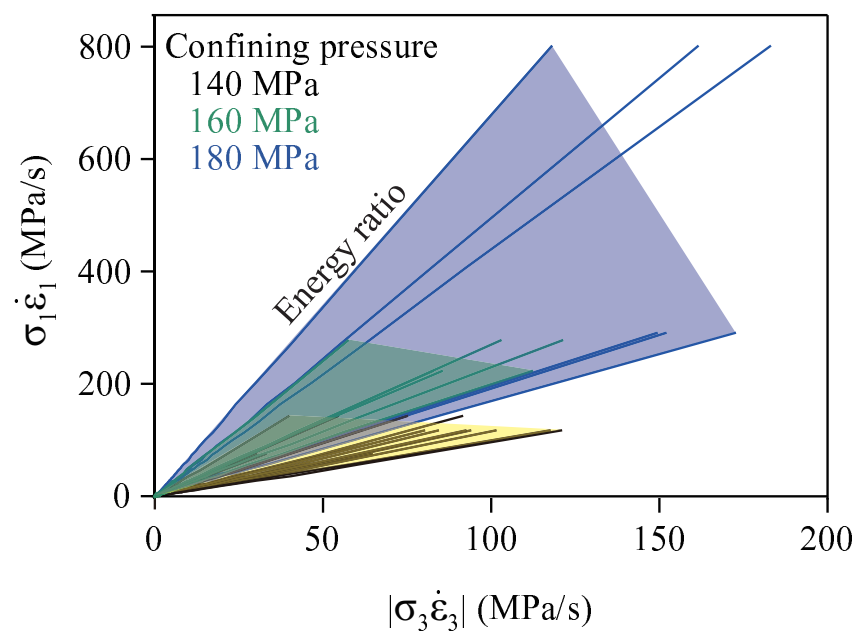

Figure 5. The ratio of the energy rate of the major principal compressive stresses to minor compressive stresses. Yellow, green and blue mean 140, 160 and $180 \mathrm{MPa}$ of confining pressure, respectively. Single line was obtained by a signal of a strain gauge at each experiment. mechanical behaviors of gouges and microstructural development. After that, we mention the relationship of the energy ratio and microstructural development.

\section{Mechanical behaviors of gouges}

Our experimental results showed three different behaviors of gouges depending on differential stresses. The contraction of gouges observed at low stress is elastic rebound. This behavior occurred without stress drop because the loading stress was still low. So, in all experiments, this behavior of gouges was commonly observed at lower stress (e.g., 1st holding in Figure 4). On the other hand, great extension at high stress means plastic deformation (4th in Figure 4). This plastic behavior implies formation of R1-shears, which appeared after peak stress (Logan et al., 1992; Marone, 1998). The difference in strain indicates microstructural heterogeneity of gouges. After this plastic behavior, the differential stress did not increase nevertheless continuous loading because plastic deformation or slip had already started. Marone et al. (1990) pointed out that compaction of gouges happens at early shear, and that unstable slip occurs after enough compaction. Thus, our results show the process until unstable slip occurs as follows; at first, gouges show elastic rebound depending on the progress of compaction. When compaction of gouges progresses fully leading to dense distribution, gouges show plastic deformation because of the formation of R1-shears. Finally, the growth from R1shear to Y-shear results in unstable slip (e.g., Logan et al., 1992).

\section{The energy ratio}

From our experimental results, it is revealed that the energy ratio 

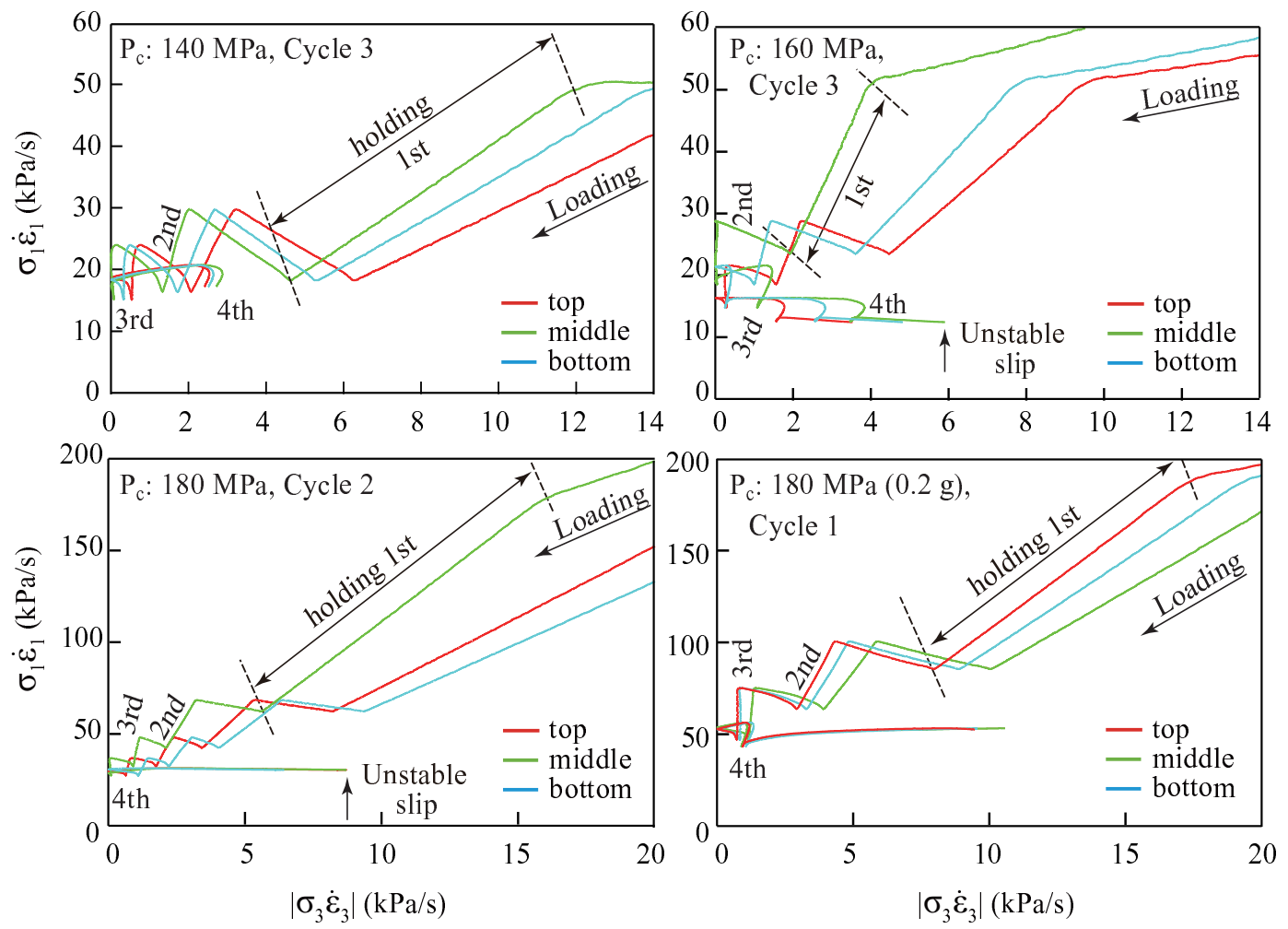

Figure 6. The enlarged diagram of Figure 5 at each experiment. The color means position of strain gauges. The remarkable change in energy ratio is shown at the final loading.

changed at each holding and loading stage, and it increased with confining pressure. Moreover, the change in the energy ratio at the final loading stage is remarkable.

The cause for the increases in the energy ratio with confining pressure is compaction. Under high confining pressure, the gouges can endure slip because they are highly dense under high confining pressure. Therefore, gouges show high strength, and the energy ratio becomes large. In the triaxial compression state, the Rowe's theory has been written as

$$
\frac{\sigma_{1}}{\sigma_{3}}=\left(1-\frac{\dot{\varepsilon}_{v}}{\dot{\varepsilon}_{1}}\right) \tan ^{2}\left(\frac{\pi}{4}+\frac{\varphi}{2}\right),
$$

where $\dot{\varepsilon}_{v}$ is the volumetric strain rate and $\varphi$ is the internal friction angle (Niiseki, 2001). Eq. (3) indicates that the volumetric strain rate is related to the stress, and so it has been called the stress-dilatancy relation, which can be derived from the critical state (Schofield and Wroth, 1968) or the variational principle of granular mechanics (Niiseki, 2001). Based on the relation: $\dot{\varepsilon}_{v}=\dot{\varepsilon}_{1}+\dot{\varepsilon}_{2}+\dot{\varepsilon}_{3}=\dot{\varepsilon}_{1}+2 \dot{\varepsilon}_{3}$, the stress ratio is rewritten as.

$$
\frac{\sigma_{1}}{\sigma_{3}}=-\frac{2 \dot{\varepsilon}_{3}}{\dot{\varepsilon}_{1}} \tan ^{2}\left(\frac{\pi}{4}+\frac{\varphi}{2}\right) .
$$

So, using Eq. (2) and Eq. (4), $K$ is given by

$$
K=-2 \tan ^{2}\left(\frac{\pi}{4}+\frac{\varphi}{2}\right)
$$

Therefore, $K$ is related to the internal friction angle $\varphi$ of the fault gouges.

Riedel shear angles related to the internal friction angle decrease with shear strain in connection with microstructural development $(\mathrm{Gu}$ and Wong, 1994). From our experiments, it is clear that the energy ratio varied at higher stress at which gouges behaved plastically (Figures 3, 4 and 6). This remarkable change at higher stress implies frictional instability and reflection of arrangements or fabrics of gouge particles. According to Rowe's law, mechanical energy is dissipated through the process of rearranging and crushing of granular materials (Rowe, 1962). Consequently, we propose the new method to assess energetic behaviors such as frictional instability using the energy ratio.

Unconsolidated materials such as fault gouges and soft sediments exist in shallower parts of active faults and accretionary wedges in subduction zones. From our experimental results, the fault gouges are proved to obey Rowe's theory of the constant energy ratio until frictional instability occurs. Moreover, it has been known that this Rowe's theory can be applied not only to granular materials but also to blocky materials (Chappell, 1979). This indicates that the Rowe's theory can be applied to seismogenesis in active faults and subduction zone plate boundaries. Based the theory of passive or active earth pressure in the soil mechanics, the internal friction angles of geomaterials like soft sediments determine the longitudinal shape of a slip surface in landslides or spray faults in accretionary prisms (e.g., Terada and Miyabe, 1929). From our experimental results (Eq. 5), the internal friction angle of geomaterials is related to the energy ratio according to Rowe's law. So, using Rowe's law and the monitoring of energy ratio (i.e., stress ratio and volumetric strain) in situ (e.g., in the borehole), we will be able to predict the longitudinal shape of a slip surface in landslides or spray faults in accretionary 
prisms. Therefore, various natural geohazards related to frictional or mechanical instability of granular and blocky geomaterials, such as earthquakes and landslides, can be evaluated with Rowe's theory of constant energy ratio.

\section{Conclusion}

We conducted 12 friction experiments using simulated fault gouges and concluded as follows. The Rowe's theory of the constant energy ratio is proved to hold for fault gouges, for instance quartz in our study. Thus, Rowe's theory can be applied to fault mechanics. The occurrence of natural disasters such as earthquakes and landslides can be assessed by Rowe's theory of constant energy ratio. These experimental observations provide us better understanding on the relationship among microstructural development, frictional instability and the energy ratio of geomaterials based on Rowe's law on seismogenic process or landslides.

\section{Acknowledgements}

We thank Professor Emeritus K. Otsuki for his assistance in experiments and many discussions. This study was funded by a Grantin-Air for Scientific Research (24244077).

\section{References}

Byerlee, J., Mjachkin, V., Summers, R., and Voevoda, O., 1978, Structures developed in fault gouge during stable sliding and stick-slip, Tectonophysics, v. 44, pp. 161-171.

Chappell, B. A., 1979, Deformational response in discontinua, International Journal of Rock Mechanics and Mining Sciences \& Geomechanics Abstracts, v. 16, pp. 377-390.

Gu, Y. and Wong, T.-f., 1994, Development of shear localization in simulated quartz gouge: Effect of cumulative slip and gouge particle size, Pure and Applied Geophysics, v. 143, pp. 387-423.

Ikari, M. J., Marone, C., and Saffer, D. M., 2011, On the relation between fault strength and frictional stability, Geology, v. 39, pp. 83-86.

Logan, J. M., Dengo, C. A., Higgs, N. G., and Wang, Z. Z., 1992, Chapter 2 Fabrics of experimental fault zones: Their development and relationship to mechanical behavior, In: Evans, B., and Wong, T.-f. (Eds), International Geophysics Series, Volume 51, Fault Mechanics and Transport Properties of Rocks. A Festschrift in Honor of W. F. Brace, Academic Press, New York, pp. 33-67.
Logan, J. M., Friedman, M., Higgs, N., Dengo, C., and Shimamoto, T., 1979, Experimental studies of simulated gouge and their application to studies of natural fault zones, Proceedings of Conference VIII, Analysis of Actual Fault Zones in Bedrock, U. S. Geol. Surv., Open File Rep., 79-1239, pp. 305-343.

Marone, C., 1998, Laboratory-derived friction laws and their application to seismic faulting, Annual Review of Earth and Planetary Sciences, v. 26, pp. 643-696.

Marone, C., and Kilgore, B., 1993, Scaling of the critical slip distance for seismic faulting with shear strain in fault zones, Nature, v. 362, pp. 618621.

Marone, C., Raleigh, C. B., and Scholz, C. H., 1990, Frictional behavior and constitutive modeling of simulated fault gouge, Journal of Geophysical Research, v. 95, pp. 7007-7025.

Marone, C., and Scholz, C. H., 1988, The depth of seismic faulting and the upper transition from stable to unstable slip regimes, Geophysical Research Letters, v. 15, pp. 621-624.

Moroto, N., 1991, Rowe's stress dilatancy theory and variational principle, Proceedings of the 26th Annual Conference of the Japan Society of Soil Mechanics and Foundation Engineering, The Japan Society of Soil Mechanics and Foundation Engineering, Tokyo, pp. 549-550 (in Japanese).

Niiseki, S., 2001, Formulation of Rowe's stress-dilatancy equation based on plane of maximum mobilization: Powders and Grains 2001, Proceedings of the Fourth International Conference on Micromechanics of Granular Media, Sendai, Japan, 21-25 May, 2001, edited by Kishino, Y., A.A. Balkema Publishers, pp. 213-216.

Onuma, K., Muto, J., Nagahama, H., and Otsuki, K., 2011, Electric potential changes associated with nucleation of stick-slip of simulated gouges, Tectonophysics, v. 502, pp. 308-314.

Reynolds, O., 1885, On the dilatancy of media composed of rigid particles in contact, Philosophical Magazine Series 5, v. 20, pp. 469-481.

Rowe, P. W., 1962, The stress-dilatancy relation for static equilibrium of an assembly of particles in contact, Proceedings of the Royal Society London A, v. 269, pp. 500-527.

Schofield, A., and Wroth, P., 1968, Critical State Soil Mechanics, McGrawHill, London, 310 pp.

Scholz, C. H., Wyss, M., and Smith, S. W., 1969, Seismic and aseismic slip on the San Andreas fault, Journal of Geophysical Research, v. 74, pp. 2049-2069.

Terada, T., and Miyabe, N., 1929, Experimental investigations of the deformation of sand mass by lateral pressure, Bulletin of the Earthquake Research Institute, University of Tokyo, v. 6, pp. 109-126.

Wong, T.-f., Baud, P., and Klein, E., 2001, Localized failure modes in a compactant porous rock, Geophysical Research Letters, v. 28, pp. 25212524 . 